\title{
Assessment of Human Exposure to Electromagnetic Fields: Review and Future Directions
}

\author{
Akimasa Hirata, Fellow, IEEE, Yinliang Diao, Member, IEEE, Teruo Onishi, Kensuke Sasaki, \\ Members, IEEE, Seunyoung Ahn, Senior Member, IEEE, Davide Colombi, Member, IEEE, Valerio \\ De Santis, Senior Member, IEEE, Ilkka Laakso, Member, IEEE, Luca Giaccone, Wout Joseph, Essam \\ A. Rashed, Senior Members, IEEE, Wolfgang Kainz, Member, IEEE, Ji Chen, Senior Member, IEEE
}

\begin{abstract}
This paper reviews recent standardization activities and scientific studies related to the assessment of human exposure to electromagnetic fields (EMF). The differences of human exposure standards and assessment of consumer products and medical applications are summarized. First, we reviewed human body modeling and tissue dielectric properties. Then, we explain the rationale of current exposure standards from the viewpoint of EMF and the standardization process for product compliance based on these exposure standards. The assessment of wireless power transfer, as an example of emerging wireless devices, and environmental EMFs in our daily lives are reviewed. Safety in magnetic resonance systems, where the EMF exposure is much larger than from typical consumer devices, is also reviewed. Finally, we summarize future research directions and research needs for EMF safety.
\end{abstract}

Index Terms - compliance assessment, computational methods, electromagnetic safety, human body modeling, medical safety

\section{INTRODUCTION}

$\mathrm{W}$ ITH the development of various electronic systems, humans are increasingly being exposed to electromagnetic fields (EMFs). International standardization bodies, the IEEE International Committee on Electromagnetic Safety (ICES) Technical Committee (TC) 95 and the International Commission on Non-Ionizing Radiation Protection (ICNIRP), set safety guidelines/standards to protect people from excessive exposure to EMF [1-3]. These guidelines prescribe exposure limits for people in restricted environments and for the general public in unrestricted environments. These

Manuscript received February 28, 2021; revised xxxx: accepted xxxx. Date of publication xxxx; date of current version xxxx. (Corresponding authors: Akimasa Hirata and Ji Chen)

Akimasa Hirata is with the Department of Electrical and Mechanical Engineering, Nagoya Institute of Technology, Nagoya 466-8555, Japan (email: ahirata@nitech.ac.jp). Yinliang Diao is with College of Electronic Engineering, South China Agricultural University, Guangzhou 510642, China (diaoyinliang@ieee.org). Teruo Onishi and Kensuke Sasaki are with National Institute of Information and Communications Technology, Tokyo 184-8795, Japan (e-mails: teruo.onishi@ieee.org, k_sasaki@nict.go.jp). Seunyoung Ahn is with Cho Chun Shik Graduate School of Green Transportation, Korea Advanced Institute of Science and Technology, Daejeon 34051, South Korea (e-mail: sahn@kaist.ac.kr). Davide Colombi is with Ericsson Research, Ericsson AB, $164 \quad 80 \quad$ Stockholm, Sweden (e-mail: davide.colombi@ericsson.com). Velerio De Santis is with the Department of exposure limits provide a high degree of protection against adverse health effects of exposure from both short- and longterm, continuous, and intermittent EMFs.

These exposure limits are not intended for patients under the direction of physicians and medical professionals. Typically, they are also not applicable with respect to the use of medical devices or implants. Exposure during medical treatment can be one or two orders of magnitude higher than the standards for the general public. In contrast to maximizing safety for the public from EMF exposure, clinical applications of EMFs must find a balance between efficacy, which typically increases with increasing EMF exposure, and safety. EMF exposure for clinical applications has a wide range of exposure strengths and frequencies. For EMF exposure limit setting, computational and experimental dosimetry are essential. Numerical and measurement methods are used to assess the compliance of wireless equipment against the limits prescribed in the guidelines or exposure standards.

There is increasing public concern regarding safety issues for new/emerging wireless systems. The World Health Organisation (WHO)'s research agenda recommends assessing exposures from new wireless network technologies [4]. After its publication, several emerging wireless systems appeared, such as the fifth generation wireless communication system $(5 \mathrm{G})$ and wireless power transfer (WPT) systems. They have led to heighten discussion regarding safety of wireless technologies, as well as

Industrial and Information Engineering and Economics, University of L'Aquila, L'Aquila, Italy (e-mail: valerio.desantis@univaq.it). Luca Giaccone is with Dipartimento Energia "G. Ferraris", Politecnico di Torino, 10129 Torino, Italy (e-mail: luca.giaccone@polito.it). Essam Rashed is with the Department of Mathematics, Faculty of Science, Suez Canal University, Ismailia 41522, Egypt (e-mail: erashed@ @science.suez.edu.eg). Ilkka Laakso is with the Department of Electrical Engineering and Automation, Aalto University, Espoo 02150, Finland (e-mail: ilkka.laakso@aalto.fi). Wout Joseph is with the Department of Information Technology (INTEC), Ghent University-IMEC, 9052 Ghent, Belgium (e-mail: Wout.Joseph@UGent.be). Wolfgang Kainz is with the Center for Devices and Radiological Health, Food and Drug Administration, Silver Spring, MD 20852, USA (e-mail: wolfgang.kainz@fda.hhs.gov). Ji Chen is with the Department of Electrical and Computer Engineering, University of Houston, Houston, TX 77204-4005, USA (e-mail: jchen23@Central.UH.EDU). 


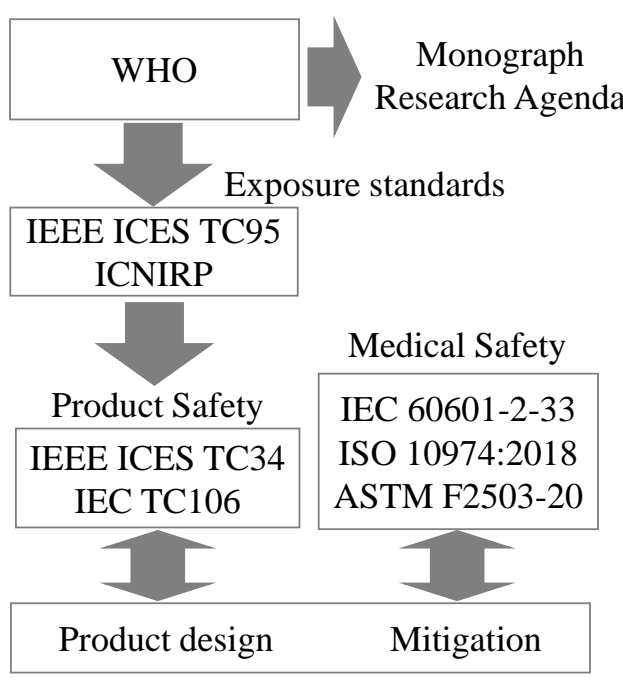

Fig. 1. Relationship between human health, exposure standard, product compliance, medical safety, and related standardization. The standards of medical equipment safety are listed as examples.

challenges in electromagnetic compatibility (EMC). For example, electric vehicles (EVs) often require large currents to support vehicle operation and can induce large EMF inside passengers (IEC PT62764-1), in addition to the WPT emissions occurring during recharging operations (see Sec. V.B). The IEEE ICES TC34 and TC106 of International Electrotechnical Commission (IEC) are responsible for the exposure assessment. Medical diagnostic devices and therapies, such as magnetic resonance imaging (MRI) and electrical stimulation, apply electromagnetic signals to humans (see Sec. VI). TCs from the IEC and the International Organization of Standardization (ISO) along with local/regional regulatory bodies or societies (e.g., American Society for Testing and Materials, ASTM) are responsible for setting the limits and test procedures for medical diagnostic devices.

Continuous improvements in computational and experimental methods are essential to develop a scientific basis for exposure standards and product compliance assessment methods. Different standardization bodies are responsible for human exposure safety, product compliance for environmental EMF, and medical devices. Figure 1 summarizes the relationship between standardization bodies. This paper covers an overview of selected topics in the field of electromagnetic safety including a brief history and future directions. The scope of this paper includes: review of the up-to-date dosimetry studies (together with human body modeling and tissue dielectric properties), which provide scientific basis for developing recent exposure standards; the standardization process for product compliance based on these exposure standards; and human safety for WPT, environmental EMFs, and MRI systems as examples. Product safety assessments, which are not currently under standardization, are out of scope of this paper.

The outline of this review is as follows. In Section II, we review human body modeling and tissue dielectric properties, essential for representing the biological body in the computational domain. In Section III, we review the rationale

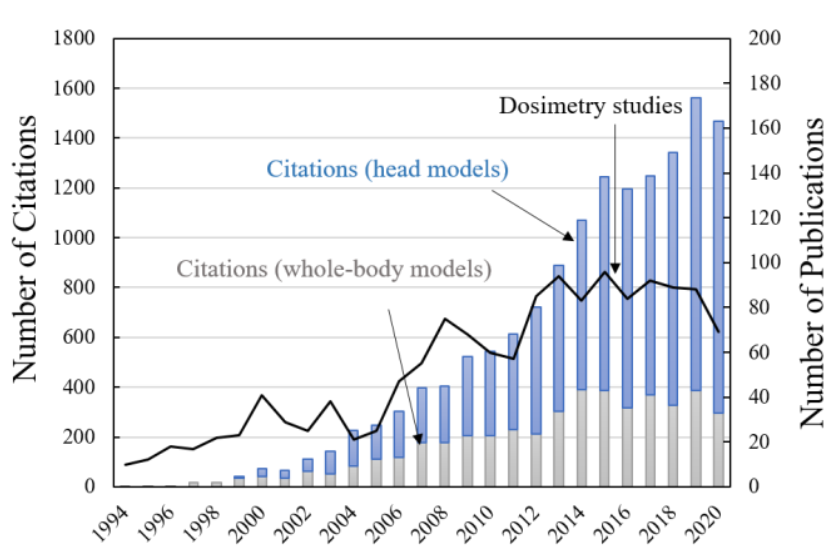

Fig. 2. Numbers of citations of whole-body and head models and that of publications on dosimetry studies (1994-2020).

for international exposure standards and guidelines from the viewpoint of electrical engineering. In Section IV, we review the assessment procedure for product compliance together with the latest updates on standardization. In Section V, we briefly discuss topics related to human safety such as emerging WPT systems and mitigation of exposure to their EMF, environmental field measurements, and related environmental health issues. In Section VI, recent trends in electromagnetic safety in biomedicine are reviewed, emphasizing on safety regarding MRI.

\section{Electromagnetic Modeling OF Human Body}

\section{A. Human body models}

Human models play a vital role in characterizing and analyzing several physical and biological effects in the research area related to EMC; such as human protection, product safety, and biomedical sciences [5]. With a long history starting with simple geometric representations [6], human models have reached an advanced quality representing human anatomy with superior accuracy (e.g., [7]). Computational models are commonly available in voxels or representations that fit different simulation platforms. Most of these models are constructed using segmentation and medical image clustering to identify different anatomical structures (such as tissues, fluids, structures, etc.).

Earlier attempts for anthropomorphic human models, as in [8] consisted of 36 organs/tissues in $1 \mathrm{~mm}$ image resolution; however, the longitudinal resolution was low at 5-10 $\mathrm{mm}$. Later, the same number of organs was presented at a unified $2 \mathrm{~mm}$ resolution [9]. Models of adult male and female subjects have been developed to represent different races (e.g., [10-13]). Moreover, a set of libraries have been developed to demonstrate models representing different ages, genders, BMI index, etc. (e.g., [14]). Special models that represent pregnant women have been constructed in $[15,16]$. Several studies have also developed infants and children models (e.g., [17]). Model postures (standing, sitting, arms up, etc.) have been customized to provide more reliable application-based models, as shown in [18, 19]. Recent developments in artificial intelligence, particularly deep learning, has led to remarkable breakthroughs in several medical 
imaging and signal processing applications [20]. In particular, it has become possible to generate reliable, high quality segmentation in a short time which is close to expert manual segmentation $[21,22]$. These techniques are expected to facilitate personalized modeling in the future.

Fig. 2 shows the trend of citation of key papers on whole-body models, in addition to head models including personalized head models. While the total number of citations is increasing citations on whole-body models have been mostly saturated the last five years. Research on personalized human head modeling has become more popular in the last five years.

\section{B. Dielectric Properties of Biological Tissue}

The dielectric properties of tissues (permittivity and conductivity) are frequency-dependent and fundamental physical quantities essential for computational dosimetry. The database in [23], mainly based on comprehensive dielectric measurements of over 50 mammal tissues at frequencies up to $20 \mathrm{GHz}$, has been widely used for computational dosimetry.

The variations in tissue dielectric properties by species (human and animal) [24, 25] and postmortem effects (in vivo and in vitro) [24, 26], from $100 \mathrm{MHz}$ to $10 \mathrm{GHz}$, have been investigated. Studies reported that the dielectric properties of mammalian tissue well represent those of humans [24]. Although the physiological and biochemical changes inside tissue after death may affect the dielectric properties, the postmortem effect has not been well characterized because of the change in tissue state (e.g., [30]). In addition, the effects of aging on the dielectric properties of tissues were investigated [24, 27], allowing the dielectric data to reflect variations in age for numerical human models.

At frequencies higher than $6 \mathrm{GHz}$, the dielectric properties of tissues comprising the skin [28, 29] and eyes have been measured [30]. Variations in the dielectric properties of skin at each body part have also been reported owing to the variation in the thickness of the stratum corneum and the underlying tissues [29]. Below $1 \mathrm{MHz}$, the electrochemical phenomenon called the electrode-polarization effect is the dominant uncertainty term. Its effect is not clearly quantified (e.g., [31]). Therefore, knowledge of dielectric properties in this frequency range is limited [30, 32].

\section{HUMAN EXPOSURE SAFETY}

International guidelines specify two types of limits $[1,2,33]$. Basic restrictions (BRs, also known as dosimetric reference limits, DRLs [1]) correspond to the physical quantities inside the exposed body. They are derived from exposure thresholds corresponding to substantiated adverse health effects by considering the reduction factors. The reference levels (RLs), also known as exposure reference levels (ERLs), are defined in terms of the electric field, magnetic field, or power density without the presence of the human body. They are derived from the BR/DRL, providing a practical method to demonstrate compliance with guidelines or standards.

Fig. 2 also shows the number of new publications for dosimetry studies with keywords including "specific absorption rate (SAR)" and/or "electric field," and "human body models or human head models." Non-related papers were excluded manually. Similar to the trend of citation of key papers on whole-body models, the number of new publications became stable in the last five years. Among applications at intermediate frequencies, WPT was selected from the number of papers published in 2015-2020, related to the dosimetry in the search (total 51 publications). Note that the keywords for latest publications becomes variable, such as "skin heating" or "nerve stimulation" making it difficult to cover all possible keywords in a single search.

\section{A. Low-frequency Dosimetry}

The dosimetric quantity used to define the BR in the low frequency (LF) range is the internal [2] (or in situ [1]) electric field. The rationale of these limits is to provide protection from adverse effects arising from stimulation of electrically excitable tissue. Because the induced electric field cannot be measured easily, it must be assessed numerically.

Dosimetry modeling at LF relies on the assumption that the electromagnetic wavelength is much longer than the human body. Hence, the system is quasistatic. Furthermore, the magnetic skin depth is much longer than the dimensions of the body due to the relatively low conductivity of biological tissue, and thus, the secondary magnetic field can be ignored. Under these assumptions, the induced electric field can be characterized by the electric scalar potential. The most commonly used computational methods at LF are the scalarpotential finite-difference method [34] and the finite-element method (FEM) [35].

The computational results are subject to numerical artifacts. Human body models are commonly discretized using regular voxels that introduce a staircasing effect in the results [36]. To mitigate the effect of numerical artifacts, the ICNIRP guidelines require that, for a specific tissue, the $99^{\text {th }}$ percentile value of the electric field is the relevant value to be compared with basic restrictions. In the case of localized exposure, the $99^{\text {th }}$ percentile can underestimate the maximum induced electric field [37]. In these cases, higher percentile values, such as the $99.9^{\text {th }}$ percentile, have been used in the literature [38]. Recently, a proposed statistically based approach removed the underestimation caused by percentile filtering [39, 40].

One of the most important sources of uncertainty in LF dosimetry is the electrical conductivity of tissues. Conventionally, tissue conductivity values have been estimated based on data from animal tissue measurements [23]. The uncertainty in the tissue properties can considerably impact the induced electric fields. For instance, the conductivity value of the skin can have a large impact on the estimation of the induced electric field [41]. Furthermore, different estimates of brain tissue conductivity can produce discrepancies of approximately one-third in the highest electric field values in the brain [42].

Before comparing the estimated electric field with the BRs or DRLs, it needs to be spatially averaged. Spatial averaging accounts for the effective distance for the electrical excitation of tissue. In ICNIRP the electric field is averaged over $2 \mathrm{~mm} \times$ $2 \mathrm{~mm} \times 2 \mathrm{~mm}$ cubic volumes [2], whereas IEEE C95.1 averages it over a $5 \mathrm{~mm}$ line segments [1]. Although limitations have previously been identified in the averaging method of ICNIRP (e.g., [43]), a recent study showed that both averaging methods produce comparable and conservative estimates of the electric 
field [44].

For non-sinusoidal waveforms, both ICNIRP and IEEE proposed a Fourier transform-based approach. The spectrum of the waveform is computed and, considering only the magnitudes, each spectral line is divided by the corresponding limit. The summation of these terms must be lower than 1 for compliance. This method can be conservative because it assumes that all spectral components are added in phase. To reduce conservativeness, ICNIRP allows the use of the weighted peak method [2]. In this method, the fields are weighted using a complex filter function whose equivalent in the time domain can be defined using analog filters. In the IEEE standard C95.1 [1], coherent non-sinusoidal waveforms can be considered by determining their frequency from the phase durations of the waveform. The limits for sinusoidal waveforms can be applied with minor modifications afterward.

The above-mentioned limits are settled based on the field strength in a conservative manner without considering the nerve stimulation, in which detailed multiscale (nerve activation) simulation is needed [45].

\section{B. Radio-frequency Dosimetry}

The dosimetric quantity used to determine the exposure in the radio-frequency (RF) range is the SAR and absorbed/ epithelial power density (i.e., the power density absorbed in tissue from an incident RF wave). At frequencies below $6 \mathrm{GHz}$, peak spatial-SAR cubically averaged over $10 \mathrm{~g}$ of tissue is an approximate surrogate of local temperature rise [46]. Above 6 $\mathrm{GHz}$, the international guidelines introduced the absorbed/ epithelial power density averaged over $4 \mathrm{~cm}^{2}$ as a surrogate for skin temperature rise [47]. Whereas IEEE C95.1 [1] limits the whole-body average SAR up to $6 \mathrm{GHz}$, the ICNIRP guidelines [33] provides limits for the whole-body averaged SAR up to $300 \mathrm{GHz}$. which is based on the study of [48], revealing that infrared radiation can increase the body core temperature.

The finite-difference time-domain (FDTD) technique and the FEM have been extensively adopted for computational electromagnetics and thermodynamics (multi-physics) in the evaluation of induced fields (BRs or DRLs) under RF exposure because of its ability to accurately model complex inhomogeneous media. Dosimetry modeling for whole-body exposure revealed that a whole-body average SAR of at least 6 $\mathrm{W} / \mathrm{kg}$, in the frequency range of $100 \mathrm{kHz}$ to $6 \mathrm{GHz}$, is required to increase the body core temperature in adults by $1{ }^{\circ} \mathrm{C}$ for exposure of at least $1 \mathrm{~h}$ [49]. The guidelines set a conservative value of $4 \mathrm{~W} / \mathrm{kg}$ for a body-core temperature rise of $1{ }^{\circ} \mathrm{C}$ after $30 \mathrm{~min}$ of exposure.

The SAR and temperature rise in the human head and body due to exposure below $6 \mathrm{GHz}$ have been widely studied for local exposure. The heating factor, or the ratio of the peak temperature rise to the peak value of SAR averaged over $10 \mathrm{~g}$ tissue, was observed to be lower than $0.25^{\circ} \mathrm{C} /(\mathrm{W} / \mathrm{kg})$ in the skin and $0.1^{\circ} \mathrm{C} /(\mathrm{W} / \mathrm{kg})$ in the brain after $30 \mathrm{~min}$ of exposure [50].

Recent studies have focused on the exposure to RF EMF above $6 \mathrm{GHz}$, the frequency range newly allocated for $5 \mathrm{G}$ communications. Owing to decreasing penetration depth with increasing frequencies, the heating effect of the EMF at this range mainly affects superficial tissues (e.g., the skin). Theoretical modeling of EM absorption and tissue heating using one-dimensional stratified models has been adopted in this frequency range [51-53]. For oblique incident waves, the absorbed power density (APD) and resulting temperature rise are functions of the angle of incident wave to the surface. The transmittance of the TM (transverse magnetic) wave into the skin increases with the angle of incidence up to the maximum transmittance angle $[53,54]$ because of Brewster's angle effect. In general, the normal incidence scenario was found to be the worst case for surface temperature rise [53].

Several studies investigated the temperature rise from brief exposures to EMF $[55,56]$. They showed that the thermal time constant declines with increasing frequency when the EM absorption localizes closer to the body surface. The maximum transient temperature rise produced by an intense pulse may exceed the steady-state temperature rise produced by continuous exposure to the same total fluence.

Computational errors resulting from the staircasing and simulation settings such as spatial resolution, source modeling, boundary condition, and stop criterion, are common uncertainties associated with RF dosimetry using the FDTD method, but can be reduced by proper implementation and using more computational resources [57]. Different SAR calculation methods may lead to variations in the results [58]. However, the numerical uncertainty at RF is usually not significant because the absorbed power (SAR or APD) is averaged over $10 \mathrm{~g}, 4 \mathrm{~cm}^{2}$ of tissue, or the whole body. In addition, the temperature distribution is smooth because of the heat diffusion and is thus insensitive to numerical uncertainties [59].

Parameter uncertainties including variations in dielectric properties, thermal properties, thermoregulatory models, and thickness of the tissue layers in the body models may also affect the calculations. The effects of dielectric properties on averaged SAR or APD are not significant; the standard deviations of SAR averaged over $10 \mathrm{~g}$ of tissue caused by $20 \%$ variations in dielectric constant and conductivity are below 15\% and decrease with increasing frequency [60]. This is also valid for frequencies from $10 \mathrm{GHz}$ to $1 \mathrm{THz}$ where the transmittance is slightly affected by variations in the tissue dielectric properties [52]. Tissue blood flow varies greatly depending on the individual and body parts. The typical variability of peak temperature rises in anatomical head models caused by variations in blood flow is less than 15\% [61]. At higher frequencies, the increase in skin temperature strongly depends on tissue thickness and the heat transfer coefficient between the skin and the environment $[51,52,62]$.

\section{MEASUREMENT METHODS AND STANDARDIZATION FOR EXPOSURE ASSESSMENT}

\section{A. Mobile Wireless Devices}

An electric field probe [63] is used for SAR measurements according to the procedure standardized by international bodies such as the IEC TC106 and IEEE ICES TC34. The latest SAR measurement procedure for portable devices have been published as IEC/IEEE 62209-1528 [64]. A phantom composed of a shell filled with a tissue-equivalent liquid is utilized because the probe can scan within the liquid easily and measure the three-dimensional electric field distribution using a robot. 
The phantom, called specific anthropomorphic mannequin (SAM) [64], has a realistic shape, produces conservative SAR estimates, and considers the device usage in the temporal region of the human head. A flat phantom is used for other parts of the body. The flat phantom was carefully investigated to obtain the conservative value when introduced. Information and rationale about the phantoms design are found in [64]. It is timeconsuming to measure the SAR using this procedure, but measurements are characterized by high repeatability and acceptable uncertainty. To overcome the time constriction a new SAR measurement method was introduced. The method consists of an electric field vector probe array to simultaneously measure the SAR in the entire plane of interest and thereby significantly reducing the measurement time. The threedimensional electric field distribution can then be estimated based on the two-dimensional SAR measurement by the array (e.g., $[64,65])$

The above-mentioned procedures do not apply to devices intended to be implanted in the human body. In general, the transmitting power for an in-body wireless devices (such as medical implant communication system) and wearable communications are low [66, 67]. If the maximum transmit power of a wireless device is at a level described in [68], measurements of SAR following [64] may be exempted.

General computation techniques to assess the SAR using the FDTD [69] and FEM [70] have also been standardized. Computational assessment of SAR for specific products including vehicle-mounted antennas [71] and mobile phones [72] have also been standardized. Pros and cons, requirements and conditions for the applicability of existing numerical methods and measurement techniques used to determine SAR for mobile wireless devices are provided in detail by the referenced standards (e.g. [69]-[72]).

Measurements of the incident power density (IPD) for terminals are recently required at frequencies above $6 \mathrm{GHz}$. The IPD can be obtained easily in the far-field region because the ratio of the electric and magnetic fields is constant. However, the evaluation of IPD becomes more complex in close proximity to radiation sources, i.e., the near-field. Currently, a few proposed methods use the Poynting vector, which requires complex electric and magnetic field measurements. A study is ongoing, in which the definition is more consistent with the IEEE ICES TC95 exposure guidelines. Unlike the SAR measurement, the IPD is measured in free space without a phantom. IEC TC106 investigated and reported the IPD measurement procedure in [73]. There are some computation techniques to obtain the Poynting vector using phase information from the measured amplitude, estimation of the magnetic field from the measured electric field (or vice versa), and back transformation (e.g., [74-76]). At lower frequencies, such as $10 \mathrm{GHz}$, the user of a wireless device operating at a distance of only a few millimeters from the body is exposed to the reactive or radiative near field. In such cases, particular considerations for conservatively assessing the exposure based on the evaluation of the IPD in free space may be necessary [77].

\section{B. Base Stations for Mobile Communications}

The RF-EMF compliance assessment of mobile base stations is normally a regulatory requirement. It typically involves establishing a so-called compliance boundary (or exclusion zone) surrounding a base station antenna outside which the EMF exposure is below the limits. Measurement methods and numerical techniques to determine the compliance boundary have been investigated, and improved over the years to keep pace with the rapid advancements in mobile technologies, as summarized in technical standards (e.g., [78]). The evaluation method to be selected depends on several factors, such as product type, operation frequency, distance from the transmitting antennas, etc. Advantages and disadvantages of each approach are documented in [78]

Although frequencies below $6 \mathrm{GHz}$ will continue to provide the backbone for mobile communications, with $5 \mathrm{G}$, the frequency range of utilization has extended up to the millimeter wave region (e.g., 5G technology currently targets frequencies between $24.25 \mathrm{GHz}$ and $52.6 \mathrm{GHz}$ ). SAR, power density, and field strength measurement methods applicable below $6 \mathrm{GHz}$ are well established; however, recent studies focused on the development of techniques covering the spectrum up to approximately $100 \mathrm{GHz}$. For base stations, compliance is typically assessed by evaluating the IPD in the far field of the transmitting antennas. Therefore, the same measurement procedures applicable below $6 \mathrm{GHz}$ also apply in the millimeter-wave region. Exposure might need to be determined in close proximity to the base station for very low-power products (small cells). In this case, near-field measurement techniques similar to those recently developed for EMF compliance assessment of wireless devices may be necessary (see Section IV.A). These methods typically are timeconsuming, especially if whole-body exposure, which requires averaging of power density over a much larger area than localized exposure, needs to be assessed. Therefore, the development of more efficient techniques is required.

$5 \mathrm{G}$ makes use of advanced antenna technologies such as massive multiple input multiple output (MIMO) allowing the base station to direct the energy where it is needed rather than constantly transmitting energy to a wide angular sector. Compared with conventional antennas, the time-averaged transmit power of MIMO antennas is spatially distributed among different beams dynamically selected by the base station to serve the users. When applied to beamforming antennas, traditional compliance evaluation methods would assume that the theoretical maximum power is transmitted in each possible beam for long periods, leading to unrealistic exposure assessments. Recently, several numerical and experimental studies determined the actual maximum power level contributing to EMF exposure for base stations implementing beamforming (e.g., [79-82]). Procedures applicable for massive MIMO base stations will be included in the next revision of IEC 62232 [78].

When conducting compliance measurements of massive MIMO base station installations directly in situ, instantaneous exposure will be characterized by a large variability of transmitted signals caused by variations in the data traffic and 
dynamic changes in the scheduled beam for transmission. Extrapolation techniques, as described in IEC 62232, are to be used (e.g., [83-85]) when the objective of the measurements is to evaluate the maximum exposure at a location on site.

\section{RECENT TOPICS RELATED TO ELECTROMAGNETIC SAFETY}

\section{A. RF Environmental Field Measurement}

Compliance assessments of mobile equipment (see Sec. IV) are conducted according to test procedures assessing the exposure levels to ensure that products meet the limits under exceptional conditions. Assessment of RF environmental EMF is needed to investigate real-life in situ exposure to EMF caused by existing and new (5G) telecommunication and broadcasting technologies. These environmental fields are not only caused by telecommunication base stations and broadcasting antennas, but also by the users (mobile) devices [86, 87].

To assess environmental RF-EMF, different measurement setups and protocols have been proposed. These can be categorized as: spot measurements using accurate spectral equipment (narrow-band measurements) or broadband meters [86, 87]; personal exposure meter (exposimeter) setups for microenvironmental (urban, rural, schools, homes, public places) or population studies (surveys) (e.g., [88]); and spatialtemporal field mapping using fixed and mobile RF sensors placed in cities [89].

Spot measurements are the most accurate ones and can be used to assess compliance to limits set by international exposure guidelines/standards [1,33]. The measurement uncertainty (expanded uncertainty with a confidence interval of 95\%) was estimated to be $\pm 3 \mathrm{~dB}$ in situ [87, 90]. The calibration of measurement probes occurs in free space or an anechoic chamber.

Personal exposimeter studies used exposimeters that measure time-varying electric field strengths in different frequency bands worn on the body [88]. These devices can be used in epidemiological and micro-environmental studies. However, they are calibrated while standing alone in an anechoic room. This lack of on-body calibration combined with body shadowing and cross-talk can cause uncertainties up to $30 \mathrm{~dB}$ [91]. This uncertainty can be resolved using on-body calibration and multiple exposimeters on the body.

Spatial-temporal field monitoring is realized with fixed RF sensors deployed in smart city networks with Internet of Things (IoT) infrastructure. This environmental monitoring with lowcost RF sensors enables the investigation of long-term field exposure and can be used for risk communication to the public. It also enables the creation of spatial maps of EMFs in real environments [92] and monitors the temporal behavior of the fields over periods spanning multiple years $[89,93]$. The RF sensors are calibrated in anechoic rooms (4-5 dB uncertainty) but in practice placed on walls of buildings, roofs, poles, etc. Variations between different RF sensors in the range 5-12 dB for different frequency bands have been observed [89].

\section{B. Exposure Assessment of WPT Systems}

WPT techniques have been investigated to recharge the batteries of various devices, including mobile electronics, home appliances, and EVs. The demand for fast charging has increased the power level of WPT systems for EVs from 3.3 up to $22 \mathrm{~kW}$ [94], yielding an EMF leakage larger than in conventional wireless systems. This leakage in the neighborhood environment has increased the need to determine compliance of WPT systems with the EMC and safety standards $[1,2,33]$. To answer this requirement, a technical report of assessment methods for the WPT systems is available [95] and its standardization is ongoing.

Besides standardization process, the exposure assessment of WPT systems has been extensively investigated in the scientific literature. Close-range or low-power portable devices have been evaluated for different types of systems and coupling mechanisms: induction coupling in the $\mathrm{kHz}$ range [96] and resonant coupling in the MHz range [97]. These studies showed that the spatial peak value of the external magnetic field is one or two orders of magnitude higher than the RL in the international guidelines, whereas the induced electric field and SAR are well within the BR. Several studies addressed human exposure to WPT systems in EVs for different human postures and chassis materials (e.g., [98, 99]). The electric field is induced in the human body by the magnetic flux crossing the body surface based on Faraday's induction law. Thus, the induced electric field and SAR are higher in the trunk rather than the remaining body parts for larger coils [100]. Other studies of importance are those on the charging of a dynamic WPT system (e.g., [101]) and safety issues related to passengers with medical implants, particularly active implantable medical devices [102].

\section{Mitigation of EMF from WPT Systems}

Some traditional EMC techniques, such as shielding, filtering, or balancing, can be used to mitigate the EMF. However, the WPT system should send some power wirelessly over a certain distance, which strictly limits the application of conventional EMC techniques. Therefore, ad hoc mitigation methods applicable to WPT systems have been proposed for various applications.

Passive shielding techniques by metallic contact, effectively reducing the magnetic field leakage, have been proposed but have significant limitations in physical implementation [103]. Different groups proposed active shielding to reduce the magnetic field leakage by field cancelation [104, 105]. A resonant reactive shielding method to compensate for the disadvantage of active shielding, such as requirement of additional current source and difficulty in synchronization between source and cancelling fields, has been presented [106]. Furthermore, reactive shielding methods are applied to the WPT system of electric vehicles [107]. Owing to the advantages of compact design and minimal degradation efficiency, the reactive shield can be applied to mobile applications reducing the magnetic field exposure to the human body [108].

As the number of medical electronic devices increase, the reduction of the magnetic field on implantable medical devices has also been suggested in [109]. Using a multi-resonant shielding method, the fundamental and harmonic frequencies were reduced, thus simultaneously considering both electromagnetic interference and EMF [110]. 
To prevent unwanted EMF from WPT systems, different aspects of research is conducted. In the international standard of the Society of Automotive Engineers (SAE) [94], sensors for detecting foreign objects are required, so that the system can reduce or turn off the power transfer in an emergency. This object detection system prevents heating of metallic objects in the vicinity of the WPT system and reduces possible interference with active implantable medical devices [111, 112].

\section{MedicAl Aspects}

Many medical procedures utilize EMFs for therapy (e.g., diathermy using high-frequency electric currents to stimulate the circulation), diagnostic applications (e.g., microwave based breast imaging used as a complimentary tool to mammography), and MRI [113].

First described experimentally in 1946 [114], MRI has become 'the' diagnostic imaging tool with over 100 million examinations performed worldwide in 2020 [115]. From the first clinical MR images in the 1980s, MRI has evolved into an essential diagnostic tool capable of capturing images of any part of the body such as the brain, joints, abdomen, and legs in any imaging direction. Although EMF exposure during MR examinations results in one of the highest whole-body RF exposures, reaching up to 50 times the environmental exposure limits for the general public, MRI has an outstandingly safe history of EMF use. Over the years, many standards and guidance documents have been developed to guide MR system manufacturers and users toward safe and effective MR systems and MR examinations [116]. To assess the EMF safety of patients during MR examinations, a critical distinction between patients with and without implants must be made.

\section{A. MR EMF Safety for Patients without Implants}

IEC 60601-2-33 [117] standardizes EMF exposure during MR examinations. Additionally, the ICNIRP published three statements pertaining to the protection of patients during MR procedures [118-120]. MRI uses a combination of a strong static magnetic field $\left(\mathrm{B}_{0}\right)$, RF pulses $\left(\mathrm{B}_{1}\right)$, and temporary changes in the magnetic or gradient field to produce a detectable $\mathrm{RF}$ signal later converted into an image. A typical MR system with $\mathrm{B}_{0}=3 \mathrm{~T}$ uses a static magnetic field 100,000 times stronger than Earth's magnetic field.

Although short-term low static magnetic field exposure has no significant effects, high static magnetic fields affect the human body adversely. Strong magnetic fields exert electrodynamic forces on moving ions in the blood vessels [121] of stationary patients, leading to the generation of electric potentials across the blood vessels and a reduction in blood flow velocity in the aorta of approximately $12 \%$ at $18 \mathrm{~T}$ [122]. However, no clinically significant effects on heart rate, respiratory rate, systolic and diastolic blood pressure, finger pulse oxygenation levels, or core body temperature of healthy volunteers, during and after exposure to an $8 \mathrm{~T}$ static magnetic field, were found [123]. Healthy human subjects, continuously moving in a strong static magnetic field of up to 8T, reported sensations of dizziness, headache, vertigo, nausea, balance problems, the perception of flickering light in the visual field, a metallic taste probably due to the electrolysis of metallic chemicals in the subjects' teeth fillings, and increased reaction time [123] all leading to an increased risk of accidental injuries [124]. For adults and children older than 1 month, the U.S. Food and Drug Administration (FDA) designates MR scanners above 8T (4T for children less than 1 month old) as a significant risk device, that is, a device presenting a potential for serious risk to the health, safety, or welfare of a subject (21 C.F.R. \$812.3), requiring an investigational device exemption (IDE) prior to conducting a clinical trial [125].

The rapidly switched gradients produce time-varying EMFs often described by the parameter $\mathrm{dB} / \mathrm{dt}$. This parameter induces EMFs in a subject in accordance with Faraday's law that, with sufficient magnitude, can lead to peripheral nerve or cardiac stimulation. Peripheral nerve stimulation can become intolerable resulting in termination of the scan. Cardiac fibrillation, which has a higher threshold than cardiac stimulation, is a life-threatening situation that must be categorically avoided. The thresholds for peripheral nerve and cardiac stimulation increase with decreasing pulse width and depend on the spatial direction of the gradient field. For very long gradient EMF pulses, the peripheral nerve stimulation threshold is $\sim 19 \mathrm{~T} / \mathrm{s}$ [126], whereas the cardiac stimulation threshold is $\sim 400 \mathrm{~T} / \mathrm{s}$ [127].

Induced currents are the primary RF absorption mechanism of $\mathrm{B}_{1}$ RF EMF exposure in the human body. The secondary RF absorption mechanism is the capacitive coupling of stray electric fields close to the matching capacitors. Stray electric fields expose the tissues in close proximity to the rungs and endrings of the RF coil. These electric fields strongly depend on the actual coil design. To ensure patient safety, IEC 60601-2-33 [117] standardizes the RF absorption by setting limits for the whole-body averaged SAR, the head-averaged SAR, and the partial-body averaged SAR for volume transmit coils. For local transmit coils, the local SAR averaged over any $10 \mathrm{~g}$ of tissue is limited. In the normal operating mode, the IEC limits are 2 $\mathrm{W} / \mathrm{kg}$ for the whole-body averaged SAR; $3.2 \mathrm{~W} / \mathrm{kg}$ for the headaveraged SAR; $2-10 \mathrm{~W} / \mathrm{kg}$ for the partial body-averaged SAR depending on the exposed body mass; $10 \mathrm{~W} / \mathrm{kg}$ for the $10 \mathrm{~g}$ averaged local SAR in the head and trunk; and $20 \mathrm{~W} / \mathrm{kg}$ in the extremities.

Both absorption mechanisms lead to localized and wholebody tissue heating which can lead to tissue damage with sufficient magnitude. Because tissue damage not only depends on the tissue temperature increase but also on the exposure time, the transient temperature increases are translated into an effective thermal load by applying the thermal dose model of cumulative equivalent minutes at $43{ }^{\circ} \mathrm{C}$ (CEM43) [128]. To prevent potential tissue damage, maximum tolerable CEM43 values and maximum allowed scan times have been established for various tissues [129].

\section{B. MR EMF Safety for Patients with Implants}

For patients with implants, the situation becomes more complicated. Implants are typically divided into passive implantable medical devices (PIMDs) and active implantable medical devices (AIMDs). For proper identification and safe 
handling, PIMDs and AIMDs are labeled either as MR Safe, MR Unsafe, or MR Conditional (allowing safe scanning under very specific conditions outlined in the device labeling) [130]. Whereas non-conductive PIMDs can often be labeled as MR Safe, conductive PIMDs are typically MR Unsafe or MR Conditional. Based on the physical nature of their interaction, conductive PIMD patient hazards in the MR environment can be grouped into three categories: forces and torques on ferromagnetic objects due to the static magnetic field $\mathrm{B}_{0}$, heating, resulting in possible tissue damage because of RFinduced currents in the implant by the time-varying RF EMF $B_{1}$ and the gradient EMF, and image artifacts, obscuring the region of interest or leading to possible misinterpretation of the image. The ASTM published standards [116] recognized by the U.S. FDA to test for the aforementioned PIMD hazards and to classify medical devices in the MR environment [130]. Labeling AIMD [131] as MR Conditional requires, in addition to the three PIMD hazard categories, testing for: gradient EMFinduced vibration; gradient and $\mathrm{B}_{1}$ EMF-induced rectified lead voltage, resulting in possible unintended stimulation; gradient, $\mathrm{B}_{0}$, and $\mathrm{B}_{1}$ EMF-induced device malfunction; and a combined field test [132]. PIMD and AIMD tests are performed experimentally, computationally, or a combination thereof [133]. Recent safety concerns include the safety of legacy PIMDs and AIMDs and the safety of broken and abandoned AIMD leads, which can result in a 20 -fold increase in RF EMFinduced heating [134-136].

\section{RESEARCH NEEDS}

The overall improvement in human model accuracy is an important topic as mentioned in Sec. II A and B. This may include an inter-subject variability study using different models or an analysis of the population level. In addition, the accurate measurement of dielectric human tissue properties is essential for evaluating the induced field strength. Recent studies suggest that the conductivity of skin and brain tissues may be significantly higher than those commonly used in LF dosimetry (e.g., [31]). Recent publications reported a novel approach for dielectric measurements that allows the estimation of dielectric human tissue properties in vivo using MRI [137]. These and other novel medical imaging and measurement technologies will help to further improve human body modeling.

Research needs in the areas of human EMF safety (Sec. III) are summarized by various international standardization bodies $[4,138,139]$. The topics listed therein relate to human body modeling, as well as the necessity of more accurate dosimetry, which includes reductions of uncertainties, and multi-physics or multi-scale methods for correlating the field quantities with substantiated biological effects (e.g., [140]). The assessments of exposure to emerging LF and RF technologies and simultaneous exposure (local and whole-body) to multiple sources will also prove useful for identifying potential changes in the dominant factors affecting EM safety. Additional research on human EM safety will provide more scientific data and improve the justifications for exposure limits, especially at frequencies higher than $6 \mathrm{GHz}$, and in the intermediate frequency range.
New mobile wireless technologies, such as $5 \mathrm{G} / 6 \mathrm{G}$ and beyond, are being developed continuously. Their safe use demands not only conservative short- and long-term exposure limits for the new frequencies, but also adequately standardized compliance methods. Therefore, additional relevant exposure evaluation methods are needed to support frequencies from $100 \mathrm{GHz}$ up to $300 \mathrm{GHz}$ within the next 10 years. As the complexity of new wireless technologies has substantially increased over the years, further development and standardization of novel and more efficient EMF compliance testing methodologies are needed. International standardization will continue to play a key role in harmonizing global test procedures to establish compliance with current and future wireless technologies. One challenge faced by standardization will be to define test conditions that provide conservative, but realistic exposure conditions based on real-life situations, rather than relying on theoretical or unreasonable assumptions and simplifications. The assessment methods by the computation may become more common for emerging wireless systems, and thus further research is needed for verification of its effectiveness in different scenarios.

Future work for the environmental spectral measurement (see Sec. V) will focus on measurement procedures to assess worstcases and realistic exposure to EMF from 5G New Radio (NR)MIMO (Ma-MIMO) with a focus on sub- $6 \mathrm{GHz}$ and millimeterwave bands. The literature scarcely documents in situ assessment of 5G Ma-MIMO signals at millimeter waves and the proper adjustment of measurement settings. For personal exposimeter studies, protocols for $5 \mathrm{G}$ personal exposure assessment will need to include active and inactive users. In contrast to legacy technologies, Ma-MIMO base station exposure strongly depends on the activity of the individual user (e.g., streaming data, downloading files, web browsing, etc.). Involving millimeter waves for personal exposure assessment is a significant challenge.

Also, in RF-MR safety it is necessary to develop clinically relevant human body models for measurements in addition to developing new computational methods for multi-scale and multi-physics applications. Most computational human body models use over 30 different types of tissue. However, below $500 \mathrm{MHz}$ dielectric human tissues properties are not significantly different from each other. Some preliminary studies provided evidence that simplified human body models can still achieve accurate EM simulation results. This leads to the possibility of developing simplified computational and experimental models for different RF-MR safety measurement needs for PIMDs, AIMDs, and high-field MR systems (e.g., [141]). Furthermore, simple and equivalent test methods/equipment those are based on the physics principles should be developed (e.g., [142]).

Acknowledgement: Main contributors of each section are as follows: Sec I (AH and JC), Sec II(KS and ER), Sec III (YD, LG, and IL), Sec IV (TO and DC), Sec V (AH, WJ, VD, and SA), Sec VI (JC and WK), and Sec VII (all authors).

Disclaimer: The mention of commercial products, their sources, or their use in connection with material reported herein is not to be construed as either an actual or implied endorsement of such products by the Department of Health and Human 
Services. The findings and conclusions in this article have not been formally disseminated by the Food and Drug Administration and should not be construed to represent any agency determination or policy.

\section{REFERENCES}

[1] W. H. Bailey et al., "Synopsis of IEEE Std C95. 1" Standard for Safety Levels With Respect to Human Exposure to Electric, Magnetic, and Electromagnetic Fields, $0 \mathrm{~Hz}$ to $300 \mathrm{GHz}$ ", IEEE Access, vol. 7, pp. 171346-171356, 2019.

[2] ICNIRP, "Guidelines for limiting exposure to time-varying electric and magnetic fields (1 Hz to $100 \mathrm{kHz}$ )," Health Phys., vol. 99, no. 6, pp. 818-836, 2010.

[3] ICNIRP, "Guidelines for limiting exposure to electrromagnetic fields ( $100 \mathrm{kHz}$ to $300 \mathrm{GHz}$ )," Health Phys., vol. 118, no. 5, pp. 483$524,2020$.

[4] WHO. "Research Agenda for Radiofrequency Fields." http://whqlibdoc.who.int/publications/2010/9789241599948 eng.p df (accessed Feb.17 2020).

[5] W. Kainz et al., "Advances in computational human phantoms and their applications in biomedical engineering - a topical review," IEEE Trans. Rad. Plasma Med. Sci., vol. 3, no. 1, pp. 1-23, 2018.

[6] H. Zaidi and X. G. Xu, "Computational anthropomorphic models of the human anatomy: the path to realistic Monte Carlo modeling in radiological sciences," Annu. Rev. Biomed. Eng., vol. 9, pp. 471$500,2007$.

[7] X. G. Xu and K. F. Eckerman, "Handbook of anatomical models for radiation dosimetry," 2009.

[8] I. G. Zubal, C. R. Harrell, E. O. Smith, Z. Rattner, G. Gindi, and P. B. Hoffer, "Computerized three- dimensional segmented human anatomy," Med. Phys., vol. 21, no. 2, pp. 299-302, 1994.

[9] P. Dimbylow, "Induced current densities from low-frequency magnetic fields in a $2 \mathrm{~mm}$ resolution, anatomically realistic model of the body," Phys. Med. Biol., vol. 43, no. 2, pp. 221-230, 1998.

[10] T. Nagaoka, S. Watanabe, K. Sakurai, E. Kunieda, M. Taki, and Y. Yamanaka, "Development of realistic high-resolution whole-body voxel models of Japanese adult males and females of average height and weight, and application of models to radio-frequency electromagnetic-field dosimetry," Phys. Med. Biol., vol. 49, no. 1, pp. 1-15, 2004

[11] C. H. Kim, S. H. Choi, J. H. Jeong, C. Lee, and M. S. Chung, "HDRK-Man: a whole-body voxel model based on high-resolution color slice images of a Korean adult male cadaver," Phys. Med. Biol., vol. 53, no. 15, pp. 4093-4106, 2008.

[12] D. Yu, M. Wang, and Q. Liu, "Development of Chinese reference man deformable surface phantom and its application to the influence of physique on electromagnetic dosimetry," Phys. Med. Biol., vol. 60, no. 17, pp. 6833-6846, 2015.

[13] J. W. Massey and A. E. Yilmaz, "AustinMan and AustinWoman: High-fidelity, anatomical voxel models developed from the VHP color images," in 38th Аnnu. Int. Conf. IEEE Eng. Med. and Biol. Soc., 2016: IEEE, pp. 3346-3349.

[14] A. Christ et al., "The Virtual Family-development of surfacebased anatomical models of two adults and two children for dosimetric simulations," Phys. Med. Biol., vol. 55, no. 2, pp. N23N38, 2010.

[15] R. Cech, N. Leitgeb, and M. Pediaditis, "Fetal exposure to low frequency electric and magnetic fields," Phys. Med. Biol., vol. 52, no. 4, pp. 879-888, 2007.

[16] T. Nagaoka, T. Togashi, K. Saito, M. Takahashi, K. Ito, and S. Watanabe, "An anatomically realistic whole-body pregnant-woman model and specific absorption rates for pregnant-woman exposure to electromagnetic plane waves from $10 \mathrm{MHz}$ to $2 \mathrm{GHz}$," Phys. Med. Biol., vol. 52, no. 22, pp. 6731-6745, 2007.

[17] E. Conil, A. Hadjem, F. Lacroux, M. F. Wong, and J. Wiart, "Variability analysis of SAR from $20 \mathrm{MHz}$ to $2.4 \mathrm{GHz}$ for different adult and child models using finite-difference time-domain," Phys. Med. Biol., vol. 53, no. 6, pp. 1511-1525, 2008.

[18] T. Nagaoka, E. Kunieda, and S. Watanabe, "Proportion-corrected scaled voxel models for Japanese children and their application to the numerical dosimetry of specific absorption rate for frequencies from $30 \mathrm{MHz}$ to $3 \mathrm{GHz}$," Phys. Med. Biol., vol. 53, no. 23, pp. 66956711,2008

[19] T. Wu et al., "Slice-based supine to standing postured deformation for Chinese anatomical models and the dosimetric results by wide band frequency electromagnetic field exposure: morphing," Rad. Protect. Dosimetry, vol. 154, no. 1, pp. 26-30, 2012.

G. Litjens et al., "A survey on deep learning in medical image analysis," Medical image analysis, vol. 42, pp. 60-88, 2017.

E. A. Rashed, J. Gomez-Tames, and A. Hirata, "Development of accurate human head models for personalized electromagnetic dosimetry using deep learning," NeuroImage, vol. 202, 2019, Art no. 116132 .

[22] L. Henschel, S. Conjeti, S. Estrada, K. Diers, B. Fischl, and M. Reuter, "Fastsurfer-a fast and accurate deep learning based neuroimaging pipeline," NeuroImage, vol. 219, 2020, Art no. 117012 .

[23] S. Gabriel, R. W. Lau, and C. Gabriel, "The dielectric properties of biological tissues: II. Measurements in the frequency range $10 \mathrm{~Hz}$ to 20 GHz," Phys. Med. Biol., vol. 41, no. 11, pp. 2251-2269, 1996.

[24] A. Peyman, S. J. Holden, S. Watts, R. Perrott, and C. Gabriel, "Dielectric properties of porcine cerebrospinal tissues at microwave frequencies: in vivo, in vitro and systematic variation with age," Phys. Med. Biol., vol. 52, no. 8, pp. 2229-2245, Apr 2007.

[25] A. P. O'Rourke et al., "Dielectric properties of human normal, malignant and cirrhotic liver tissue: in vivo and ex vivo measurements from 0.5 to $20 \mathrm{GHz}$ using a precision open-ended coaxial probe," Phys. Med. Biol., vol. 52, no. 15, pp. 4707-4719, 2007.

[26] G. Schmid, R. Ueberbacher, T. Samaras, M. Tschabitscher, and P. R. Mazal, "The dielectric properties of human pineal gland tissue and RF absorption due to wireless communication devices in the frequency range 400-1850 MHz," Phys. Med. Biol., vol. 52, no. 17, pp. 5457-5468, 2007.

[27] G. Schmid and R. Überbacher, "Age dependence of dielectric properties of bovine brain and ocular tissues in the frequency range of $400 \mathrm{MHz}$ to $18 \mathrm{GHz}$," Phys. Med. Biol., vol. 50, no. 19, pp. 47114720, 2005.

[28] E. Pickwell, B. Cole, A. Fitzgerald, M. Pepper, and V. Wallace, "In vivo study of human skin using pulsed terahertz radiation," Phys. Med. Biol., vol. 49, no. 9, pp. 1595-1607, 2004.

[29] S. Alekseev and M. Ziskin, "Human skin permittivity determined by millimeter wave reflection measurements," Bioelectromagn., vol. 28, no. 5, pp. 331-339, 2007.

[30] K. Sasaki et al., "Dielectric property measurement of ocular tissues up to $110 \mathrm{GHz}$ using $1 \mathrm{~mm}$ coaxial sensor," Phys. Med. Biol., vol. 60, no. 16, pp. 6273-6288, 2015.

[31] K. Wake, K. Sasaki, and S. Watanabe, "Conductivities of epidermis, dermis, and subcutaneous tissue at intermediate frequencies," Phys. Med. Biol., vol. 61, no. 12, p. 4376, 2016.

[32] C. Gabriel, A. Peyman, and E. Grant, "Electrical conductivity of tissue at frequencies below $1 \mathrm{MHz}$, " Phys. Med. Biol., vol. 54, no. 16, pp. 4863-4878, 2009.

[33] ICNIRP, "Principles for Non-Ionizing Radiation Protection," Health Phys., vol. 118, no. 5, pp. 477-482, 2020.

[34] T. W. Dawson and M. A. Stuchly, "High-resolution organ dosimetry for human exposure to low-frequency magnetic fields," IEEE Trans Mag., vol. 34, no. 3, pp. 708-718, 1998.

[35] W. Wang and S. R. Eisenberg, "A three-dimensional finite element method for computing magnetically induced currents in tissues," IEEE Trans. Mag., vol. 30, no. 6, pp. 5015-5023, 1994.

[36] T. Dawson, M. Potter, and M. Stuchly, "Evaluation of modeling accuracy of power frequency field interactions with the human body," Appl. Comput. Electromagnet. Soc. J., vol. 16, no. 2, pp. 162$172,2001$.

[37] I. Laakso and A. Hirata, "Reducing the staircasing error in computational dosimetry of low-frequency electromagnetic fields," Phys. Med. Biol., vol. 57, no. 4, pp. N25-N34, 2012.

[38] B. Kos, B. Valič, D. Miklavčič, T. Kotnik, and P. Gajšek, "Pre-and post-natal exposure of children to EMF generated by domestic induction cookers," Phys. Med. Biol., vol. 56, no. 19, pp. 6149-6160, 2011.

[39] J. Gomez-Tames, I. Laakso, Y. Haba, A. Hirata, D. Poljak, and K. Yamazaki, "Computational Artifacts of the In Situ Electric Field in Anatomical Models Exposed to Low-Frequency Magnetic Field," 
IEEE Trans. Electromagnet. Compat., vol. 60, no. 3, pp. 589-597, 2018.

[40] A. Arduino et al., "Accuracy assessment of numerical dosimetry for the evaluation of human exposure to electric vehicle inductive charging systems," IEEE Trans. Electromagnet. Compat., vol. 62, no. 5, pp. 1939-1950, 2020.

[41] G. Schmid, S. Cecil, and R. Überbacher, "The role of skin conductivity in a low frequency exposure assessment for peripheral nerve tissue according to the ICNIRP 2010 guidelines," Phys. Med. Biol., vol. 58, no. 13, pp. 4703-4716, 2013.

[42] M. Soldati and I. Laakso, "Effect of electrical conductivity uncertainty in the assessment of the electric fields induced in the brain by exposure to uniform magnetic fields at $50 \mathrm{~Hz}$," IEEE Access, vol. 8, pp. 222297-222309, 2020.

[43] J. Bakker et al., "Children and adults exposed to low-frequency magnetic fields at the ICNIRP reference levels: theoretical assessment of the induced electric fields," Physics in Medicine \& Biology, vol. 57, no. 7, p. 1815, 2012.

[44] Y. Diao, J. Gomez-Tames, E. A. Rashed, R. Kavet, and A. Hirata, "Spatial Averaging Schemes of In Situ Electric Field for LowFrequency Magnetic Field Exposures," IEEE Access, vol. 7, pp. 184320-184331, 2019.

[45] J. P. Reilly, "Survey of numerical electrostimulation models," Phys. Med. Biol., vol. 61, no. 12, pp. 4346-4363, 2016.

[46] A. Hirata and O. Fujiwara, "The correlation between mass-averaged SAR and temperature elevation in the human head model exposed to RF near-fields from 1 to $6 \mathrm{GHz}, "$ Phys. Med. Biol., vol. 54, no. 23, p. 7227, 2009.

[47] Y. Hashimoto et al., "On the averaging area for incident power density for human exposure limits at frequencies over $6 \mathrm{GHz}, "$ Phys. Med. Biol., vol. 62, no. 8, pp. 3124-3138, 2017.

[48] T. Brockow, A. Wagner, A. Franke, M. Offenbächer, and K. L. Resch, "A randomized controlled trial on the effectiveness of mild water-filtered near infrared whole-body hyperthermia as an adjunct to a standard multimodal rehabilitation in the treatment of fibromyalgia," Clin. J. Pain, vol. 23, no. 1, pp. 67-75, 2007.

[49] A. Hirata, T. Asano, and O. Fujiwara, "FDTD analysis of human body-core temperature elevation due to RF far-field energy prescribed in the ICNIRP guidelines," Phys. Med. Biol., vol. 52, no. 16, pp. 5013-5023, 2007.

[50] K. R. Foster, M. C. Ziskin, Q. Balzano, and G. Bit-Babik, "Modeling tissue heating from exposure to radiofrequency energy and relevance of tissue heating to exposure limits: heating factor," Health Phys., vol. 115, no. 2, pp. 295-307, 2018.

[51] M. C. Ziskin, S. I. Alekseev, K. R. Foster, and Q. Balzano, "Tissue models for RF exposure evaluation at frequencies above $6 \mathrm{GHz}$," Bioelectromagn., vol. 39, no. 3, pp. 173-189, 2018.

[52] K. Sasaki, M. Mizuno, K. Wake, and S. Watanabe, "Monte Carlo simulations of skin exposure to electromagnetic field from $10 \mathrm{GHz}$ to 1 THz," Phys. Med. Biol., vol. 62, no. 17, pp. 6993-7010, 2017.

[53] K. Li, K. Sasaki, S. Watanabe, and H. Shirai, "Relationship between power density and surface temperature elevation for human skin exposure to electromagnetic waves with oblique incidence angle from $6 \mathrm{GHz}$ to $1 \mathrm{THz}$," Phys. Med. Biol., vol. 64, no. 6, 2019, Art no. 065016.

[54] T. Nakae, D. Funahashi, J. Higashiyama, T. Onishi, and A. Hirata, "Skin temperature elevation for incident power densities from dipole Arrays at $28 \mathrm{GHz}$," IEEE Access, vol. 8, pp. 26863 - 26871, 2020.

[55] I. Laakso, R. Morimoto, J. Heinonen, K. Jokela, and A. Hirata, "Human exposure to pulsed fields in the frequency range from 6 to 100 GHz," Phys. Med. Biol., vol. 62, no. 17, pp. 6980-6992, 2017.

[56] S. Kodera and A. Hirata, "Permissible SA and Radiant Exposure for Brief Exposure in GHz Region," in Int. Symp. Electromagn. Compat., 2019: IEEE, pp. 1020-1024.

[57] I. Laakso, S. Ilvonen, and T. Uusitupa, "Performance of convolutional PML absorbing boundary conditions in finitedifference time-domain SAR calculations," Phys. Med. Biol., vol. 52, no. 23, pp. 7183-7192, 2007.

[58] I. Laakso, T. Uusitupa, and S. Ilvonen, "Comparison of SAR calculation algorithms for the finite-difference time-domain method," Phys. Med. Biol., vol. 55, no. 15, pp. N421-N4231, 2010.
$[60]$

[65] K. Kiminami, T. Iyama, T. Onishi, and S. Uebayashi, "Novel specific absorption rate (SAR) estimation method based on 2-D scanned electric fields," IEEE Trans. Electromagn. Compat., vol. 50, no. 4, pp. 828-836, 2008.

[66] M. N. Islam and M. R. Yuce, "Review of medical implant communication system (MICS) band and network," Ict Express, vol. 2, no. 4, pp. 188-194, 2016.

[67] D. Panescu, "Emerging technologies [wireless communication systems for implantable medical devices]," IEEE Engineering in Medicine and Biology Magazine, vol. 27, no. 2, pp. 96-101, 2008.

[68] Assessment of the compliance of low-power electronic and electrical equipment with the basic restrictions related to human exposure to electromagnetic fields (10 MHz to $300 \mathrm{GHz}$ ), IEC 62479, Geneva, 2010.

[69] Determining the peak spatial-average specific absorption rate (SAR) in the human body from wireless communications devices, 30 $\mathrm{MHz}$ to $6 \mathrm{GHz}$ - Part 1: General requirements for using the finite difference time-domain (FDTD) method for SAR calculations, IEC/IEEE 62704-1, 2017.

[70] Determining the peak spatial-average specific absorption rate $(S A R)$ in the human body from wireless communication devices, 30 $\mathrm{MHz}$ to $6 \mathrm{GHz}$ - Part 4: General requirements for using the finite element method for SAR calculations, IEC/IEEE 62704-4, 2020.

[71] Determining the peak spatial-average specific absorption rate (SAR) in the human body from wireless communications devices, 30 $\mathrm{MHz}$ to $6 \mathrm{GHz}$ - Part 2: Specific requirements for finite difference time domain (FDTD) modelling of exposure from vehicle mounted antennas, IEC/IEEE 62704-2, 2017.

[72] Determining the peak spatial-average specific absorption rate (SAR) in the human body from wireless communications devices, 30 $\mathrm{MHz}$ to $6 \mathrm{GHz}$ - Part 3: Specific requirements for using the finite difference time domain (FDTD) method for SAR calculations of mobile phones, IEC/IEEE 62704-2, 2017.

[73] Measurement procedure for the evaluation of power density related to human exposure to radio frequency fields from wireless communication devices operating between $6 \mathrm{GHz}$ and $100 \mathrm{GHz}$, IEC TR63170, 2018.

[74] S. Pfeifer et al., "Total field reconstruction in the near field using pseudo-vector E-field measurements," IEEE Trans. Electromagn. Compat., vol. 61, no. 2, pp. 476-486, 2018.

[75] K. Sasaki, K. Li, J. Chakarothai, T. Iyama, T. Onishi, and S. Watanabe, "Error analysis of a near-field reconstruction technique based on plane wave spectrum expansion for power density assessment above $6 \mathrm{GHz}, "$ IEEE Access, vol. 7, pp. 11591-11598, 2019.

[76] J. Lundgren, J. Helander, M. Gustafsson, D. Sjoberg, B. Xu, and D. Colombi, "A near-field measurement and calibration technique: Radio-frequency electromagnetic field exposure assessment of millimeter-wave 5G devices," IEEE Antennas Propag. Mag., 2020. [77] Assessment of power density of human exposure to radio frequency fields from wireless devices in close proximity to the head and body 
(Frequency range of $6 \mathrm{GHz}$ to $300 \mathrm{GHz}$ ) Part 1: Measurement procedure, IEC/IEEE 63195-1 FDIS, 2021.

[78] Determination of RF field strength, power density and SAR in the vicinity of radiocommunication base stations for the purpose of evaluating human exposure, IEC 62232, 2017.

[79] B. Thors, A. Furuskär, D. Colombi, and C. Törnevik, "Timeaveraged realistic maximum power levels for the assessment of radio frequency exposure for $5 \mathrm{G}$ radio base stations using massive MIMO," IEEE Access, vol. 5, pp. 19711-19719, 2017.

[80] D. Pinchera, M. Migliore, and F. Schettino, "Compliance Boundaries of 5G Massive MIMO Radio Base Stations: A Statistical Approach," IEEE Access, vol. 8, pp. 182787-182800, 2020.

[81] S. Shikhantsov et al., "Ray-tracing-based numerical assessment of the spatiotemporal duty cycle of 5G massive MIMO in an outdoor urban environment," Appl. Sci., vol. 10, no. 21, 2020, Art no. 7631. R. Werner, P. Knipe, and S. Iskra, "A comparison between measured and computed assessments of the RF exposure compliance boundary of an in-situ radio base station massive MIMO antenna," IEEE Access, vol. 7, pp. 170682-170689, 2019.

[83] H. Keller, "On the assessment of human exposure to electromagnetic fields transmitted by $5 \mathrm{G}$ NR base stations," Health Phys., vol. 117, no. 5, pp. 541-545, 2019.

[84] S. Aerts et al., "In-situ measurement methodology for the assessment of $5 \mathrm{G}$ NR massive MIMO base station exposure at sub$6 \mathrm{GHz}$ frequencies," IEEE Access, vol. 7, pp. 184658-184667, 2019.

[85] S. Adda et al., "A theoretical and experimental investigation on the measurement of the electromagnetic field level radiated by $5 \mathrm{G}$ base stations," IEEE Access, vol. 8, pp. 101448-101463, 2020.

[86] G. Dürrenberger, J. Fröhlich, M. Röösli, and M.-O. Mattsson, "EMF monitoring-concepts, activities, gaps and options," Int. J. Environment. Res. \& Public Health, vol. 11, no. 9, pp. 9460-9479, 2014.

[87] W. Joseph, L. Verloock, F. Goeminne, G. Vermeeren, and L. Martens, "In situ LTE exposure of the general public: Characterization and extrapolation," Bioelectromagn., vol. 33, no. 6, pp. 466-475, 2012.

[88] M. Röösli et al., "Conduct of a personal radiofrequency electromagnetic field measurement study: proposed study protocol," Environmental Health, vol. 9, no. 1, p. 23, 2010.

[89] S. Aerts, J. Wiart, L. Martens, and W. Joseph, "Assessment of longterm spatio-temporal radiofrequency electromagnetic field exposure," Environmental Res., vol. 161, pp. 136-143, 2018.

[90] Determination of RF field strength, power density and SAR in the vicinity of radiocommunication base stations for the purpose of evaluating human exposure, IEC/IEEE 62237-2017, 2017.

[91] J. F. Bolte, "Lessons learnt on biases and uncertainties in personal exposure measurement surveys of radiofrequency electromagnetic fields with exposimeters," Environ. Int., vol. 94, pp. 724-735, 2016.

[92] S. Aerts, D. Deschrijver, L. Verloock, T. Dhaene, L. Martens, and W. Joseph, "Assessment of outdoor RF-EMF exposure through hotspot localization using kriging-based sequential sampling," Environ. Res., vol. 126, pp. 184-191, 2013.

[93] L. Diez, R. Agüero, and L. Muñoz, "Electromagnetic field assessment as a smart city service: The smartsantander use-case," Sensors, vol. 17, no. 6, 2017, Art no. 1250.

[94] Wireless Power Transfer for Light-Duty Plug-In/Electric Vehicles and Alignment Methodology, SAE J2954., 2016.

[95] Assessment methods of the human exposure to electric and magnetic fields from wireless power transfer systems - Models, instrumentation, measurement and numerical methods and procedures (frequency range of $1 \mathrm{kHz}$ to $30 \mathrm{MHz}$ ), IEC PAS 63184, 2021.

[96] T. Sunohara, A. Hirata, I. Laakso, and T. Onishi, "Analysis of in situ electric field and specific absorption rate in human models for wireless power transfer system with induction coupling," Phys. Med. Biol., vol. 59, no. 14, p. 3721, 2014.

[97] X. L. Chen et al., "Human exposure to close-range resonant wireless power transfer systems as a function of design parameters," IEEE Trans. Electromagnet. Compat., vol. 56, no. 5, pp. 1027-1034, 2014.

[98] K. Miwa, T. Takenaka, and A. Hirata, "Electromagnetic dosimetry and compliance for wireless power transfer systems in vehicles," IEEE Trans. Electromagn. Compat., vol. 61, no. 6, pp. 2024-2030, 2019.

[99] V. De Santis, T. Campi, S. Cruciani, I. Laakso, and M. Feliziani, "Assessment of the induced electric fields in a carbon-fiber electrical vehicle equipped with a wireless power transfer system," Energies, vol. 11, no. 3, p. 684, 2018.

[100] T. Shimamoto, I. Laakso, and A. Hirata, "In-situ electric field in human body model in different postures for wireless power transfer system in an electrical vehicle," Phys. Med. Biol., vol. 60, no. 1, p. $163,2014$.

[101] V. Cirimele, F. Freschi, L. Giaccone, L. Pichon, and M. Repetto, "Human exposure assessment in dynamic inductive power transfer for automotive applications," IEEE Trans. Magn., vol. 53, no. 6, 2017, Art no. 5000304.

[102] I. A. Shah, Y. Cho, and H. Yoo, "Safety Evaluation of Medical Implants in the Human Body for a Wireless Power Transfer System in an Electric Vehicle," IEEE Transactions on Electromagnetic Compatibility, 2020.

[103] S. Ahn et al., "Low frequency electromagnetic field reduction techniques for the on-line electric vehicle (OLEV)," in IEEE Int. Symp. Electromagn. Compat., 2010: IEEE, pp. 625-630.

[104] S. Y. Choi, B. W. Gu, S. W. Lee, W. Y. Lee, J. Huh, and C. T. Rim, "Generalized active EMF cancel methods for wireless electric vehicles," IEEE Trans. Power Electron., vol. 29, no. 11, pp. 57705783, 2013.

[105] S. Cruciani, T. Campi, F. Maradei, and M. Feliziani, "Active shielding design for wireless power transfer systems," IEEE Trans. Electromagn. Compat., vol. 61, no. 6, pp. 1953-1960, 2019.

[106] S. Kim, H.-H. Park, J. Kim, J. Kim, and S. Ahn, "Design and analysis of a resonant reactive shield for a wireless power electric vehicle," IEEE Trans. Microw. Theory Tech., vol. 62, no. 4, pp. 1057-1066, 2014.

[107] J. Kim et al., "Coil design and shielding methods for a magnetic resonant wireless power transfer system," Proc. IEEE, vol. 101, no. 6, pp. 1332-1342, 2013.

[108] J. Park et al., "A resonant reactive shielding for planar wireless power transfer system in smartphone application," IEEE Trans. Electromagnetic Compat., vol. 59, no. 2, pp. 695-703, 2017.

[109] T. Campi, S. Cruciani, F. Maradei, A. Montalto, F. Musumeci, and M. Feliziani, "EMI in a cardiac implantable electronic device (CIED) by the wireless powering of a left ventricular assist device (LVAD)," IEEE Trans. Electromagnet. Compat., 2021 (in press9.

[110] J. Park et al., "Planar multiresonance reactive shield for reducing electromagnetic interference in portable wireless power charging application," Appl. Phys. Lett., vol. 114, no. 20, 2019, Art no. 203902.

[111] J. Xia et al., "Foreign object detection for electric vehicle wireless charging," Electronics, vol. 9, no. 5, 2020, Art no. 805.

[112] S. Y. Chu and A.-T. Avestruz, "Electromagnetic model-based foreign object detection for wireless power transfer," in Workshop on Control and Model. for Power Electronics, 2019: IEEE, pp. 1-8.

[113] M.-O. Mattsson and M. Simkó, "Emerging medical applications based on non-ionizing electromagnetic fields from $0 \mathrm{~Hz}$ to $10 \mathrm{THz}$," Med. Dev., vol. 12, p. 347, 2019.

[114] E. M. Purcell, H. C. Torrey, and R. V. Pound, "Resonance absorption by nuclear magnetic moments in a solid," Phys. Rev., vol. 69, no. 1-2, p. 37, 1946.

[115] Organisation for Economic Co-operation and Development (OECD). "Health Care Utilisation: Diagnostic Exams: Magnetic Resonance Imaging Exams, Total." https://stats.oecd.org/ (accessed Aug. 10, 2021).

[116] W. Kainz, F. G. Shellock, I. Crues, J. V. , and A. M. Karacozoff, Eds. MRI standards and guidance documents from the United States, Food and Drug Administration (MRI Bioeffects, Safety, and Patient Management, ch. 28.). Playa Del Rey, CA, USA: Biomed. Res. Pub. Gr., 2020.

[117] Medical electrical equipment - Part 2-33: Particular requirements for the basic safety and essential performance of magnetic resonance equipment for medical diagnosis, IEC 60601-2-33, 2015. ICNIRP, "Medical magnetic resonance (MR) procedures: protection of patients," Health Phys., vol. 87, no. 2, pp. 197-216, 2004.

[119] ICNIRP, "Amendment to the ICNIRP "Statement on medical magnetic resonance (MR) procedures: protection of patients"," Health Phys., vol. 97, no. 3, pp. 259-261, 2009.

[120] ICNIRP, "ICNIRP statement on diagnostic devices using nonionizing radiation: existing regulations and potential health risks," Health Phys., vol. 112, no. 3, pp. 305-321, 2017. 
[121] W. Kainz et al., "Development and validation of a magnetohydrodynamic solver for blood flow analysis," Phys. Med. Biol., vol. 55, no. 23, pp. 7253-7261, 2010.

[122] A. Kyriakou et al., "Patient-specific simulations and measurements of the magneto-hemodynamic effect in human primary vessels," Physiological Meas., vol. 33, no. 2, pp. 117-130, 2012.

[123] D. W. Chakeres, A. Kangarlu, H. Boudoulas, and D. C. Young, "Effect of static magnetic field exposure of up to 8 Tesla on sequential human vital sign measurements," J. Magnet. Reson. Imag., vol. 18, no. 3, pp. 346-352, 2003.

[124] A. Ghadimi-Moghadam et al., "Does exposure to static magnetic fields generated by magnetic resonance imaging scanners raise safety problems for personnel?," J. Biomed. Phys. Eng., vol. 8, no. 3, pp. 333-336, 2018.

[125] Criteria for Significant Risk Investigations of Magnetic Resonance Diagnostic Devices, Guidance for Industry and Food and Drug Administration Staff, FDA, 2014.

[126] J. A. Nyenhuis, J. D. Bourland, A. V. Kildishev, D. J. Schaefer, and F. Shellock, "Health effects and safety of intense gradient fields," in Magnetic Resonance Procedures: Health effects and safety, F. G. Schellock Ed. New York: CRC Press, 2001, ch. 2, pp. 31-53.

[127] J. P. Reilly, "Principles of nerve and heart excitation by timevarying magnetic fields," Annals of the New York Academy of Sciences, vol. 649, no. 1, pp. 96-117, 1992.

[128] S. A. Sapareto and W. C. Dewey, "Thermal dose determination in cancer therapy," Int. J. Radiat. Oncology, vol. 10, no. 6, pp. 787800, 1984.

[129] P. S. Yarmolenko et al., "Thresholds for thermal damage to normal tissues: an update," Int. J. Hyperthermia, vol. 27, no. 4, pp. 320-343, 2011.

[130] Standard Practice for Marking Medical Devices and Other Items for Safety in the Magnetic Resonance Environment, ASTM F250320, 2020.

[131] Assessment of the safety of magnetic resonance imaging for patients with an active implantable medical device, ISO/TS 10974:2018(E), 2018.

[132] Assessment of the safety of magnetic resonance imaging for patients with an active implantable medical device, International Organization for Standardization (ISO), 2018.

[133] S. Feng, R. Qiang, W. Kainz, and J. Chen, "A technique to evaluate MRI-induced electric fields at the ends of practical implanted lead," IEEE Trans. Mcrow. Theory Tech., vol. 63, no. 1, pp. 305-313, 2014.

[134] A. Yao, T. Goren, T. Samaras, N. Kuster, and W. Kainz, "Radiofrequency-induced heating of broken and abandoned implant leads during magnetic resonance examinations," Magn. Reson. Med., (to be published).

[135] Q. Zeng et al., "Investigation of RF-induced heating near interventional catheters at 1.5 T MRI: A combined modeling and experimental study," IEEE Trans. Electromagn. Compat., vol. 61, no. 5, pp. 1423-1431, 2018.

[136] R. Guo et al., "Computational and experimental investigation of RF - induced heating for multiple orthopedic implants," Magn. Reson. Med., vol. 82, no. 5, pp. 1848-1858, 2019.

[137] E. A. Rashed, Y. Diao, and A. Hirata, "Learning-based estimation of dielectric properties and tissue density in head models for personalized radio-frequency dosimetry," Phys. Med. Biol., vol. 65, no. 6, 2020, Art no. 065001 .

[138] J. P. Reilly and A. Hirata, "Low-frequency electrical dosimetry: research agenda of the IEEE International Committee on Electromagnetic Safety," Phys. Med. Biol., vol. 59, pp. R138-R149, 2016.

[139] ICNIRP, "Gaps in knowledge relevant to the "Guidelines for Limiting Exposure to Time-Varying Electric and Magnetic Fields (1 Hz-100 kHz)"," Health Phys., vol. 118, no. 5, pp. 533-542, 2020.

[140] J. Gomez-Tames et al., "Brain cortical stimulation thresholds to different magnetic field sources exposures at intermediate frequencies," IEEE Trans. Electromagn. Compat., vol. 61, no. 6, pp. 1944-1952, 2019.

[141] M. H. de Buck, P. Jezzard, H. Jeong, and A. T. Hess, "An investigation into the minimum number of tissue groups required for $7 \mathrm{~T}$ in-silico parallel transmit electromagnetic safety simulations in the human head," Magn. Reson. Med., vol. 85, no. 2, pp. 1114-1122, 2021.

[142] S. Song et al., "Dual-frequency high-electric-field generator for MRI safety testing of passive implantable medical devices," IEEE Trans. Microw. Theory Tech., vol. 68, no. 12, pp. 5423-5431, 2020. 